\title{
A numerical analysis of metrological properties of Venturi tube in the air-coal particle mixture flow measurement in power industry
}

\author{
Grzegorz Borsuk ${ }^{1 *}$, Bolesław Dobrowolski ${ }^{1}$, and Jacek Wydrych ${ }^{1}$ \\ ${ }^{1}$ Opole University of Technology, Mikolajczyka 5, Opole, Poland
}

\begin{abstract}
The main topic of the study is to evaluate the effect of particle concentration and fractional composition on the differential pressure in the Venturi tube during the measurement of flow rate of the gasparticle mixture. Gas phase flow was described by Euler method, while particles movement by Lagrange method. Calculations were performed using ANSYS Fluent Package. A series of numerical calculations were carried out on the particle loading ratio $Y \leq 2$ and their diameters of $10 \mu \mathrm{m} \leq d_{p} \leq 200 \mu \mathrm{m}$. It has been found that, except for the $Y$ concentration loading ratio, the increase in the differential pressure in the venturi tube also affects the Stokes number. It depends on the flow conditions and particles diameter. A correction function has been proposed to determine the effect of particles diameter on the results of the mixture flow measurement. The results of the calculations were compared with the Lee and Crowe experimental data. A good comparison of calculation results to measurement results was found.
\end{abstract}

\section{Introduction}

Gas-solid particles mixture flow occurs in many industries where pneumatic conveying is used. As an example power plant industry, food industry, building materials industry and many others. Proper processing and energy conversion processes require a current knowledge of the mass flow or one of its components. This problem is particularly important in the power plant industry, where the uniform supply of burners requires the measurement of the particle coal conveyed by the gas stream. For multiphase flow measurements, a videogrammetry method seems useful, which may help to identify flow regimes [1].

One of the methods of measuring homogeneous mixture flow is Venturi tube flowmeter. It has many advantages and high accuracy when the requirements specified in the relevant normative documents are fulfilled [2]. As a result of the tests, it has been found that selected types of Venturi tubes can be used to measure mixtures flow, but the presence of an additional phase changes the characteristics of the flowmeter. The condition of using a flow meter for the measurement of gas-particle mixture flow is to calibrate it in the conditions similar to those for further use.

The purpose of this paper is to indicate the possibility of applying mathematical modelling and numerical simulation to the initial evaluation of its metrological properties during diluted gas-particle mixture flow measuring. Particular studies have been made for Venturi tube, which is often used in two-phase mixture flow measuring systems, especially in pipe system in the conventional power plant $[3,4]$.

\section{Venturi flow meters in the gas-particle flow measurement systems}

Operations on the use of gas-solid mixture flow measurements were undertaken mainly for the needs of the power industry, and more precisely - pneumatic conveying of pulverized materials such a coal dust. In 1948 Carlson and others [5] proposed a dual-reducing system, consisting of a serial ASME nozzle and an orifice. The pressure measurement on the orifice, according to authors practically independent of the solid phase presence, was the basis for calculating the gas mass flow. The pressure drop in the nozzle was used to determine the mass flow of the mixture. As Teisseyre's later studies $[4,6]$ show, the Carlson method is not suitable for continuous measurements due to the gradual wear of the orifice and the dependence of the orifice differential pressure on the particle concentration in the mixture.

Fabar [7] implemented the first systematic study on the Venturi tube, created with the recommendations of the ISA standard. The layout of the measuring system, also used in works Antikajn [8] and Szatil [9, 10] is shown in Fig. 1a. As a result of the study, Fabar stated the linear dependence of the pressure drop $\Delta p_{t p}^{I}$ at the two-phase flow from the particle concentration in the mixture:

$$
\Delta p_{t p}^{I}=\left(1+E_{I} Y\right) \Delta p_{g}^{I}
$$

where:

\footnotetext{
*Corresponding author: g.borsuk@po.opole.pl
} 


$$
Y=\frac{M_{s}}{M_{g}}
$$

is loading ratio of the particles. The value $\Delta p_{g}^{I}$ corresponds to the differential pressure connected with the pure gas flow with the mass flow $M_{g}$, and $E_{I}$ is the Gästerstädt number [11]. Equation (1) is used to calculate the particle concentration in the gas, assuming that the gas mass flow is known at the two-phase flow.

The Farbar method requires an additional measurement of the gas mass flow, which in essence is a two-way method. Szatil [10] has confirmed the suitability of a Venturi tube for the measurement of gas-particle mixtures and found a weak dependence of constant $E_{I}$ on the solid phase concentration and gas velocity

Modification of the measurement method based on the Venturi tube, Graczyk presented $[12,13]$ in the form of the so-called three-signal method (Fig. 1a). This method was also used in the works of Teisseyre $[4,6]$, Leroch [14], Barth [15], Jung [16, 17], Szatil [10] and finally Payne and Crowe [18]. In addition to the pressure drop measurement $\Delta p_{t p}^{I}$ (Fig. 1a), Graczyk proposed the pressure drop measurement $\Delta p_{t p}^{I I}$ for the whole Venturi tube. As a result of the research, this author stated that one of the important problems is the selection of proper measurement points. $\Delta p_{t p}^{I I}$ to provide maximum measurement sensitivity and obtain a linear dependence of the pressure drop from the particle concentration

$$
\Delta p_{t p}^{I I}=\left(1+E_{I I} Y\right) \Delta p_{g}^{I I}
$$

Pressure drop ratio is a basis for calculating the particle concentration $\mathrm{Y}$ and the mass flow of both phases according to the formula:

$$
\pi_{t p}=\Delta p_{t p}^{I I} / \Delta p_{t p}^{I}
$$

Further, using a single Venturi tube as a mass flow meter simplifies the measurement installation in a significant degree. Graczyk studies have provided the basis for the practical application of the three-signal method.

Jung's research has made a great contribution to the development of Venturi tube [16, 17]. Jung proposed modifying the three-signal method by using a nozzle with extended cylindrical part (Fig. 1b). The pressure drop combined with the flow of the mixture through the tapered cylindrical part was the basis for the calculation of the particle concentration. According to Jung, the differential pressure $\Delta p_{t p}^{I}$ measured in the nozzle depends only on the flow of pure gas and can be the basis for calculating the gas mass flow. With the knowledge $\Delta p_{t p}^{I I}$ it is possible to calculate $Y$ and the particle mass flow. The disadvantage of the Jung method is that the influence of the presence of particles on the value $\Delta p_{t p}^{I}$ is neglected, which affects the measurement accuracy. Barth's work [15, 19] uses a different approach, by placing the measuring points at the end part of the Venturi tube (Fig. 1c).

a)

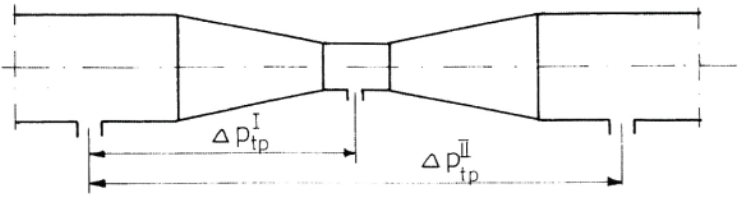

b)

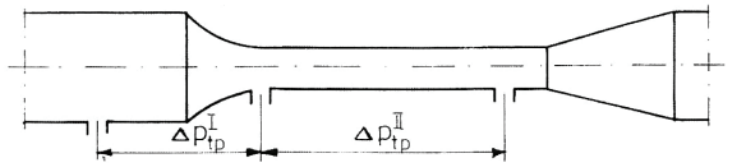

c)

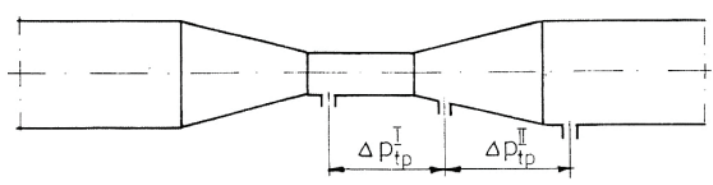

d)
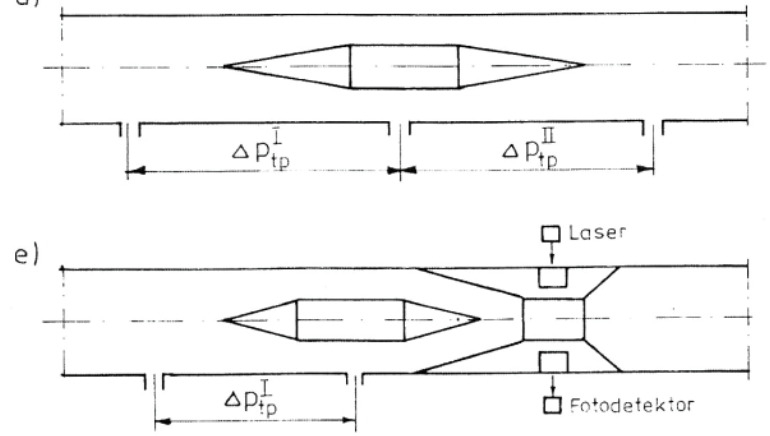

Fig. 1. Selected Venturi tube systems (described in the text).

Łapa $[14,20]$ has developed the concept of the Graczyk and has conducted detailed experiments on the venturi in the triple signal system. The study was conducted for the particle-air mixture. The effect of extensive empirical studies was to determine the influence of the pipe diameter and the venturi module on the Gästerstadt number value. Łapa has determined the calibration characteristics of the test specimens of Venturi tube by using the three-signal method to pipe diameters $\mathrm{D}<100 \mathrm{~mm}$ at $\mathrm{Y}<2$. The accuracy of the measurement method was estimated at $\pm 8 \%$.

For the local measurement of the gas-coal dust mixture concentration conveyed to the burners, the three-signal Venturi tube was used by Leroch [21]. As a result of experimental research, he defined the diameter and tube module to measure the actual velocity of both phases and particle concentration.

At the end of the 1970s, extensive studies on the evaluation of metrological properties of annular Venturi tube were undertaken in works [18, 22, 23, 24, 25] (Fig. 1d). In work [24] a measurement system based on the optical method of solid phase concentration measurement was proposed (Fig. 1e). Research on the development of measurement methods for two-phase mixtures are time-consuming and costly, so in this paper, it was indicated the possibility of supporting them with simulation methods. 


\section{Calculation methodology}

The gas-particle mixture flow is described by differential equations which can be written in the generalized conservative form, with the isolation of convection, diffusion and source components [26]:

$$
\frac{\partial(\rho \phi)}{\partial t}+\frac{\partial\left(\rho u_{i} \phi\right)}{\partial x_{i}}=\frac{\partial}{\partial x_{i}}\left(\Gamma_{\phi} \frac{\partial \phi}{\partial x_{i}}\right)+S_{\phi}+S_{\phi t}
$$

In Equation (5) $\phi$ is generalized dependent variable, $\Gamma_{\text {}}$ the diffusion coefficient. The source component will include the other components of differential equations apart from convective and diffusion. Coefficients $\Gamma_{\bullet}$ i $S_{\text {, }}$ are presented in Table 1.

Table 1. Coefficients of equation (5).

\begin{tabular}{|l|c|c|c|c|}
\hline Equation & $\phi$ & $\Gamma_{\mathrm{p}}$ & $\mathrm{S}_{\mathrm{p}}$ & $\mathrm{S}_{\mathrm{pp}}$ \\
\hline Continuity & 1 & 0 & 0 & 0 \\
\hline $\begin{array}{l}\text { Momentu } \\
\mathrm{m} \\
\text { equation } \\
\text { in the } \mathrm{x}_{\mathrm{i}} \\
\text { axis }\end{array}$ & $\mathrm{u}_{\mathrm{i}}$ & $\mu_{\mathrm{ef}}$ & $\mathrm{F}_{\mathrm{i}}-\frac{\partial \mathrm{p}}{\partial \mathrm{x}_{\mathrm{i}}}+\frac{\partial}{\partial \mathrm{x}_{\mathrm{j}}}\left(\mu_{\mathrm{ef}} \frac{\partial \mathrm{u}_{\mathrm{j}}}{\partial \mathrm{x}_{\mathrm{i}}}\right)$ & $\overline{\mathrm{S}_{\mathrm{ui}, \mathrm{p}}}$ \\
\hline $\begin{array}{l}\text { Kinetic } \\
\text { turbulenc } \\
\text { e energy }\end{array}$ & $\mathrm{k}$ & $\frac{\mu_{\mathrm{ef}}}{\sigma_{\mathrm{k}}}$ & $\mathrm{G}_{\mathrm{k}}-\rho \varepsilon$ & 0 \\
\hline $\begin{array}{l}\text { Turbulent } \\
\text { energy } \\
\text { dissipatio } \\
\mathrm{n} \text { rate }\end{array}$ & $\varepsilon$ & $\frac{\mu_{\mathrm{ef}}}{\sigma_{\varepsilon}}$ & $\frac{\varepsilon}{\mathrm{k}}\left(\mathrm{C}_{1} \mathrm{G}_{\mathrm{k}}-\mathrm{C}_{2} \rho \varepsilon\right)$ & 0 \\
\hline
\end{tabular}

$$
\begin{gathered}
\text { where: } \mu_{\mathrm{ef}}=\mu+\mu_{\mathrm{t}}, \mu_{\mathrm{t}}=\mathrm{C}_{\mu} \rho \frac{\mathrm{k}^{2}}{\varepsilon}, \\
\mathrm{G}_{\mathrm{k}}=\frac{\partial \mathrm{u}_{\mathrm{i}}}{\partial \mathrm{x}_{\mathrm{j}}} \mu_{\mathrm{ef}}\left(\frac{\partial \mathrm{u}_{\mathrm{i}}}{\partial \mathrm{x}_{\mathrm{j}}}+\frac{\partial \mathrm{u}_{\mathrm{j}}}{\partial \mathrm{x}_{\mathrm{i}}}\right) \\
\sigma_{\mathrm{k}}=1.00 \sigma_{\varepsilon}=1.30 \mathrm{C}_{1}=1.44 \mathrm{C}_{2}=1.92 \mathrm{C}_{\mu}=0.09
\end{gathered}
$$

Conversion of momentum between the diluted and continuous phase is described by the source term:

$$
\overline{S_{u i, p}}=\frac{1}{V_{E}} \eta_{j} \int_{\delta t_{j}} \frac{C_{D} \operatorname{Re}_{p} D_{p}}{8 \pi}\left(\bar{u}_{j}-u_{p i}\right) d t
$$

where $V_{E}$ is the elementary cell volume and $\eta_{j}$ describe the number of particles transported at j-trajectory in the time unit.

The particle motion was described by the Lagrange method by adding drag coefficient and gravitation:

$$
\frac{d \vec{u}_{p}}{d t}=\frac{1}{\tau_{p}}\left(\vec{u}-\vec{u}_{p}\right)+\vec{g}
$$

where $\vec{u}_{p}$ is the particle velocity vector and $\tau_{p}$ is the time of dynamic particle relaxation. As a result of the integration of equation (7) in the gas velocity field, trajectories of particle fractions were obtained. Knowledge of the trajectories is the basis for determining interphase interactions according to equation (6). Details of calculations can be found in [26]. ANSYS Fluent program [27] was used for numerical research.

\section{Numerical research results}

\subsection{Boundary conditions of the research}

The purpose of the study is to evaluate the degree of non-equilibrium flow phenomena on the metrological properties of the venturi tube in the dispersed flow. In particular, the calculations are carried out to set the connection between empirical constants in onedimensional mathematical models and the Stokes number.

In this calculation, a Venturi tube with diameter ratio $\beta=\frac{d}{D}=0.5$ was used in a pipe with a diameter $\mathrm{D}=100 \mathrm{~mm}$. The shape of the venturi tube is shown in Fig. 2, and its dimensions are given in Table 2.

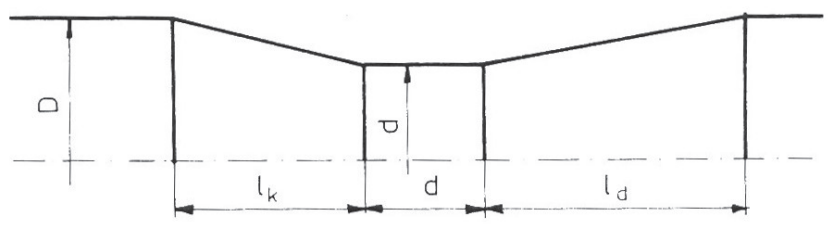

Fig. 2. Scheme of flow system with Venturi tube.

Table 2. Dimensions of Venturi tube.

\begin{tabular}{|c|c|c|c|c|}
\hline $\mathrm{D}[\mathrm{mm}]$ & $\mathrm{m}=(\mathrm{d} / \mathrm{D})^{2}$ & $\mathrm{~d}[\mathrm{~mm}]$ & $1_{\mathrm{k}}[\mathrm{mm}]$ & $1_{\mathrm{d}}[\mathrm{mm}]$ \\
\hline 100 & 0.25 & 50 & 135 & 409.8 \\
\hline
\end{tabular}

Calculations were made for a range of Reynolds number $\operatorname{Re}=5 \cdot 10^{4}-5 \cdot 10^{5}$, with gas density of $\rho_{g}=1.2 \mathrm{~kg} / \mathrm{m}^{3}$, particles density of $\rho_{s}=1400 \mathrm{~kg} / \mathrm{m}^{3}$ and their diameter from $10 \mu \mathrm{m}$ to $200 \mu \mathrm{m}$. The effect of particle concentration on differential pressure $\Delta p_{t p}$ at the two-phase flow was also tested. The range of research was proper for the concentration characteristic in pneumatic conveying in a pipe system for coal particle burned in power plants $(Y \leq 2)[3,4]$.

In the calculations, the influence of gravitation was omitted, which simplified the problem to twodimensional axial-symmetrical flow. In the inlet, a fully developed turbulent flow was assumed. Fig. 3 shows the results of the pure gas test calculations, the symbol $p_{0}$ describe pressure value in the inlet cross-section.

The increase a gas velocity, as well as, the Reynolds number $\mathrm{Re}$ cause a significant increase the differential pressure $\Delta p_{g}$. The gas mass flow is calculated from the formula: 


$$
M_{g}=\frac{C}{\sqrt{1-\beta^{4}}} \pi \frac{d^{2}}{4} \sqrt{2 \Delta p_{g} \rho_{g}}
$$

where flow coefficient is

$$
C=4 \frac{M_{g}}{d^{2}} \frac{1}{\pi} \frac{\sqrt{1-\beta^{4}}}{\sqrt{2 \Delta p_{g} \rho_{g}}}
$$

Flow coefficients calculated from the formula (9) were compared to the corresponding values according to the standard[2]. Differences do not exceed $\pm 2 \%$, which is a proof of correctness of accepted calculation methodology.

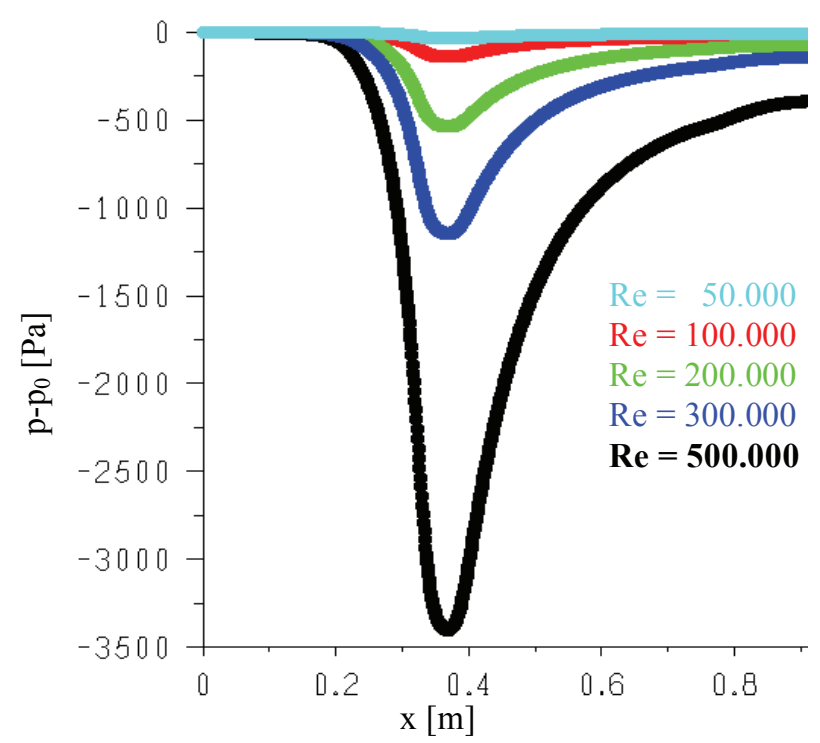

Fig. 3. Pressure distribution along the Venturi tube for different values of Reynolds number.

\subsection{Calculation results for gas-particle mixture flow}

A series of calculations were made for gas-particle mixtures. Fig. 4 shows pressure distribution along the Venturi tube for particle diameter $d_{p}=10 \mu \mathrm{m}$ with Reynolds number $\operatorname{Re}=5 \cdot 10^{5}$ for different $\mathrm{Y}$ concentrations.

Similarly, results are shown in Fig. 5. Comparison, the smaller particle diameter creates a much larger pressure drop than for diameter $d_{p}=200 \mu \mathrm{m}$ at the same particle concentration.

The results of further calculations are shown in Fig. 6. The pressure difference measured on the venturi tube is the linear function of the particle flow ratio $\mathrm{Y}$.

The angle of the characteristic inclination $\frac{\Delta p_{t p}}{\Delta p_{g}}=$ $f(Y)$ depends to a significant extent on the particle diameter, from Stokes number St defined as follows:

$$
S t=\frac{\rho_{s} d_{p}{ }^{2} U}{18 \mu_{g} D}=\frac{1}{18} \frac{\rho_{s}}{\rho_{g}}\left(\frac{d_{p}}{D}\right)^{2} R e_{G}
$$

In this case, Stokes number is in the range of 0.0324 to 129.630 .

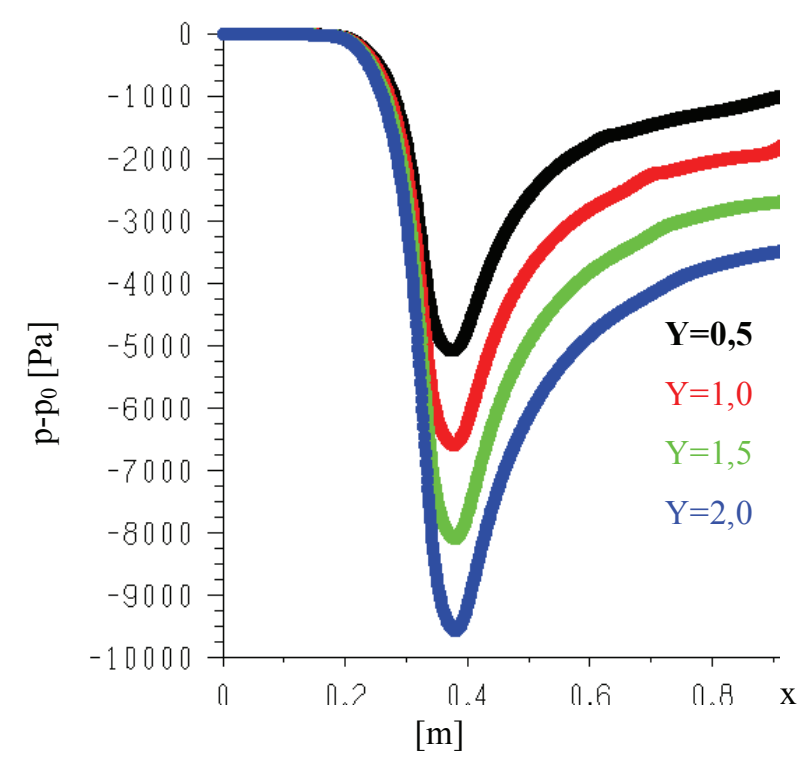

Fig. 4. Pressure distribution along the Venturi tube for $\mathrm{Re}=500000, d_{p}=10 \mu \mathrm{m}$.

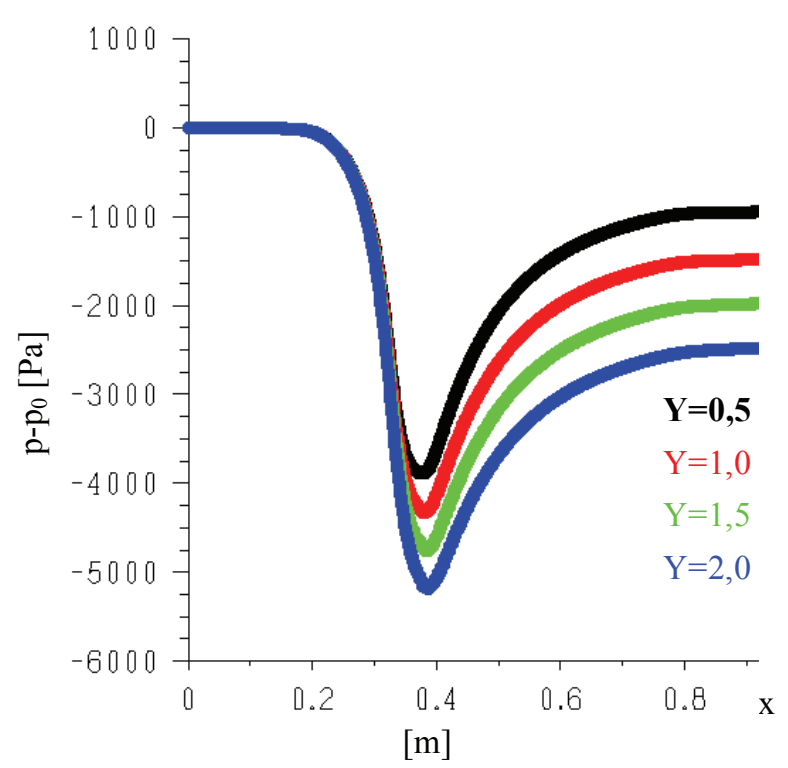

Fig. 5. Pressure distribution along Venturi tube for $\mathrm{Re}=500000, d_{p}=200 \mu \mathrm{m}$.

When the particle diameter $d_{p}$ is large, corresponding to $\mathrm{St} \rightarrow \infty$, then $\Delta p_{t p} \cong \Delta p_{g}$ and the Venturi tube practically does not react to the presence of particles. Otherwise, when $\mathrm{St} \rightarrow 0$, differential pressure $\Delta p_{t p} \cong \Delta p_{h}$ corresponds to the flow of a homogeneous mixture with a modified density. 


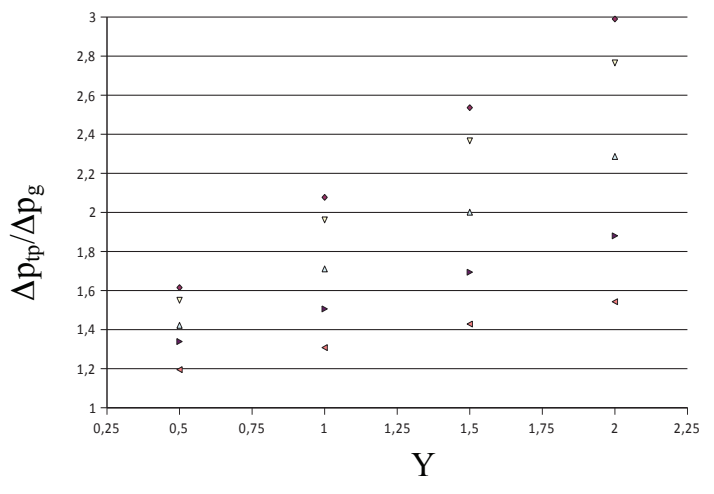

Fig. 6. Influence of particles loading ratio $Y$ on differential pressure $\Delta p_{t p}$ for different particle diameters.

Generally, the differential pressure measured on the Venturi tube is contained in the range

$$
\Delta p_{h} \geq \Delta p_{t p} \geq \Delta p_{g}
$$

So, if the Stokes number is not taken into account, the differential pressure $\Delta p_{t p}$ does not give more precise information about the mixture mass flow. The results of a wider analysis of this problem show good agreement with numerical calculations results after the introduction of the correction function $f(\Omega)$, which is equivalent to Gästerstädt number $E_{I}$ in equation (1). Then, the differential pressure $\Delta p_{t p}^{I}$ is calculated from the formula:

$$
\Delta p_{t p}^{I}=(1+f(\Omega) Y) \Delta p_{g}^{I}
$$

where

$$
f(\Omega)=\left(1+a \Omega^{b}\right)^{-1}
$$

and

$$
\Omega=\left(\frac{d_{p}}{D}\right)^{2} \frac{\rho_{s}}{\rho_{g}} R e_{g} \beta
$$

Values $a$ and $b$ in formula (13) are empirical constants depending on the fractional composition of the particles and other quantities characterizing the measurement conditions.

The function $f(\Omega)$ fulfills the asymptotic conditions:

$$
\begin{aligned}
& \lim _{\Omega \rightarrow 0} f(\Omega)=1 \\
& \lim _{\Omega \rightarrow \infty} f(\Omega)=0
\end{aligned}
$$

and ensures the fulfillment of the relation (11).

An example of the selection of empirical constants $a$ and $b$ is presented in Fig. 7, for the experimental data Lee and Crowe [23]. From the points distribution, it follows that the function $f(\Omega)$ good describes empirical data in a wide range of Stokes values.

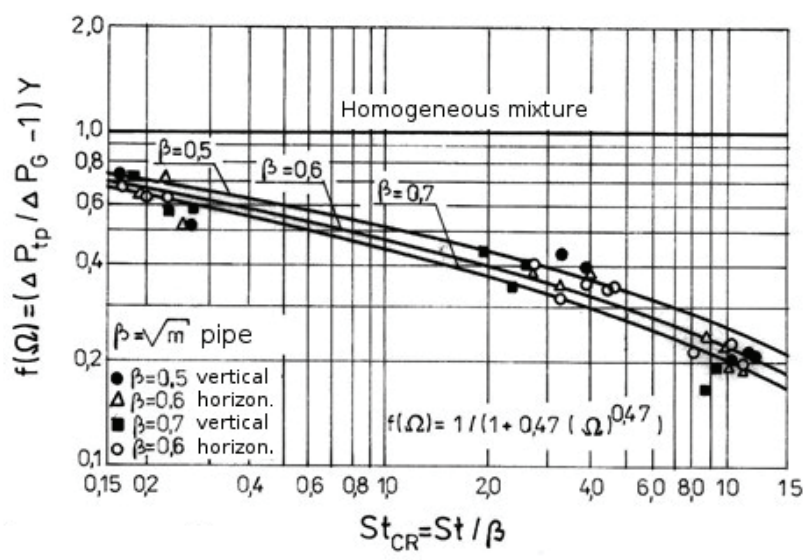

Fig. 7. The correction function $f(\Omega)$ compared with experimental data Lee and Crowe [16], where $a=b=0.47$.

Inserting $\Delta p_{g}$ from equation (12) into equation (8) we obtain an equation for calculating the two-phase mixture mass flow $M_{t p}$ :

$$
M_{t p}=\frac{C}{\sqrt{1-\beta^{4}}} \pi \frac{d^{2}}{4} \sqrt{\frac{2 \Delta p_{t p}^{I} \rho_{g}}{1+f(\Omega) Y}}
$$

where function $f(\Omega)$ is defined by equation (13). It follows that determining the mass flow of the gasparticle mixture requires, in addition to the particle concentration, also the knowledge of constant values $\mathrm{a}$ and $\mathrm{b}$ of a function $f(\Omega)$.

\section{Conclusions}

On the basis of provided analyses, the following conclusions can be drawn regarding the use of the gasparticle mass flowmeter:

1. Differential pressure $\Delta p_{t p}$ measured on the Venturi tube is caused by the flow of both phases, but in general it, does not correspond to the mass flow of the two-phase mixture.

2. When the Stokes number St $\Rightarrow 0$, the differential pressure measured on the venturi corresponds to the twophase mixture flow which can be treated as a modified density homogeneous fluid.

3. For large Stokes number, especially at $\mathrm{St} \rightarrow \infty$, the presence of particles in the gas do not have a significant effect on the differential pressure increasing, which practically corresponds to the flow of pure gas itself.

4. With a given Stokes number, the pressure increase due to the presence of the dispersion phase is a linear function of particle loading ratio.

5. The results of the measurement of the mixture mass flow or one of its components will always be erroneous if the Stokes number value is not taken into account. 


\section{References}

1. S. Anweiler, J. Environ. Manage. (2016) (https://doi.org/10.1016/j.jenvman.2017.03.040)

2. PN-EN ISO 5167-1: Measurement of particle flow ratio using measuring nozzles, Book of Polish Normalisation Committee, Warszawa, (2005)

3. D. Giddings, B. J. Azzopardi, A. Aroussi, Pickering SiJ. Powder Technol. 172, 149-156 (2007)

4. M. Teisseyre, M. Mazur, Supply of pulverized coal fired boilers - measurement issues, Book of Wroclaw University of Technology, Wrocław (2006)

5. H. M. Carlson, P. M. Frazier, R. B. Engdahl, Trans. ASME 70, 65-79 (1948)

6. M. Teisseyre, Books of Wroclaw University of Technology, Energetyka III 143, 111-136 (1966)

7. L. Farbar, Trans. ASME 75, 943-951 (1953)

8. P. A. Antikajn, Teploenergetika 12, 35-37 (1956)

9. A. A. Szatil, Tieploenergetika 8, 44-48 (1957)

10. A. A. Szatil, Izmieritelnaja Technika 9, 46-48 (1961)

11. E. Gasterstädt, Zeitschrift des Vereines Deutcher Ingenieure 24, 617-624 (1924)

12. Cz. Graczyk, Ph.D. Thesis, Silesian Univerisity of Technology, Gliwice (1957)

13. Cz. Graczyk, Pomiary Automatyka Kontrola 12, 509-511 (1970)

14. J. Łapa, Measurement of the air-particle mixture flow ratio by three-signals Venturi tube - possibilities and range of application, Books of IMA i BUT, Wroclaw University of Technology, 5, 37-50 (1971)

15. W. Barth, Chem. Ing. Tech. 3, 164-170 (1960)

16. R. Jung, Beiträge angenwandter Strömungsforschung zur Entwicklung Kohlenstaubfeuerung. Book VDI-Forschungsheft, 532 (1969)

17. R. Jung, Brennst.-Warme-Kraft 8, 377-383 (1966)

18. A. L. Payne, C. T. Crowe, Proc. 1981, Symp. Instrum. and Control Fossil Energy Processes, ANL81-62 (1981)

19. W. Barth, R. Nagel, K. van Wavern, Chem. Ing. Tech. 9, 599-602 (1957)

20. J. Łapa, Pomiary Automatyka Kontrola 2, 68-70 (1974)

21. R. Leroch, Energetyka 7/8, 27-30 (1975)

22. J. Gołąbek, Ph.D. Thesis, Silesian University of Technology, Gliwice (1979)

23. J. Lee, C. T. Crowe, J. Fluids Eng. 104, 88-91 (1982)

24. A. Payne, M. Werner, D. Plank, C. T. Crowe, The 1982 Symposium on Controls and Instrumentation of Fossil Energy Systems, Huston, June (1982)

25. L. M. Werner, C. T. Crowe, Proc. Symposium on Instrumentation and Control for Fossil Energy Processes, San Francisco, 8-9 June (1981).

26. B. Dobrowolski, J. Wydrych, JTAM 45, 513-537 (2007)

27. ANSYS Fluent, Ansys Inc. (2016) 